\title{
Nonequilibrium Dynamical Mean-Field Theory: An Auxiliary Quantum Master Equation Approach
}

\author{
Enrico Arrigoni, ${ }^{*}$ Michael Knap, and Wolfgang von der Linden \\ Institute of Theoretical and Computational Physics, Graz University of Technology, 8010 Graz, Austria
}

(Received 10 November 2012; published 20 February 2013)

\begin{abstract}
We introduce a versatile method to compute electronic steady-state properties of strongly correlated extended quantum systems out of equilibrium. The approach is based on dynamical mean-field theory (DMFT), in which the original system is mapped onto an auxiliary nonequilibrium impurity problem imbedded in a Markovian environment. The steady-state Green's function of the auxiliary system is solved by full diagonalization of the corresponding Lindblad equation. The approach can be regarded as the nontrivial extension of the exact-diagonalization-based DMFT to the nonequilibrium case. As a first application, we consider an interacting Hubbard layer attached to two metallic leads and present results for the steady-state current and the nonequilibrium density of states.
\end{abstract}

DOI: 10.1103/PhysRevLett.110.086403

PACS numbers: 71.27.+a, 05.60.Gg, 47.70.Nd, 73.40.-c

Due to the progress made in microscopically controlling quantum mechanical systems within quantum optics and ultracold quantum gases [1-4], in solid-state nanoscience, spintronics, molecular electronics, [5,6] as well as ultrafast laser spectroscopy [7-10], the interest in correlated systems out of equilibrium has steadily increased in recent years. These achievements have prompted new and boosted old related theoretical questions such as nonequilibrium quantum phase transitions [11], dissipation and decoherence [12], and thermalization after a quantum quench [13-15].

In this respect, the theoretical description and understanding of strongly correlated quantum systems out of equilibrium poses an exciting challenge to modern theoretical physics. A widely used and successful method to treat strongly correlated lattice systems in equilibrium is dynamical mean-field theory (DMFT) [16-18]. The success of the method lies on the one hand in the nontrivial treatment of dynamical properties and on the other hand in its applicability to a range of different problems, from solid-state fermionic systems to ultracold bosonic atoms, as well as the possibility to combine it with realistic electronic structure methods [19]. Recently, DMFT has been extended to deal with time-dependent nonequilibrium problems [20-25]. The extensions are based on the Kadanoff-Baym-Keldysh nonequilibrium Green's function approach [26-29].

DMFT relies on the solution of a correlated impurity problem that constitutes the bottleneck of the approach. Several techniques have been adopted in the equilibrium case. Most of them have been applied, in a more or less

Published by the American Physical Society under the terms of the Creative Commons Attribution 3.0 License. Further distribution of this work must maintain attribution to the author(s) and the published article's title, journal citation, and DOI. approximate or limited way, to nonequilibrium DMFT as well, either in a steady state or within full timedependence. Among them are the iterated perturbation theory [20], the numerical renormalization group [23], continuous time quantum monte carlo (CTQMC) calculations [24,30], noncrossing approximation, and beyond [31]. Additionally, exact DMFT solutions are available in certain limits $[21,32,33]$. Nonequilibrium quantum impurity problems (not within DMFT) have also been studied by means of scattering-state approaches [34,35]; perturbative methods [36,37] in combination with the renormalization group (RG) $[38,39]$, the time-dependent density-matrix RG [40,41], and the numerical RG [42] flow equation [43]; the functional RG [44,45]; dual fermions [46]; and finally CTQMC calculations on an auxiliary system with an imaginary bias [47-49].

In this Letter, we propose an approach that, in contrast to previous work, while directly accessing steady-state properties, features a solution of the DMFT impurity problem with controlled accuracy. This means that the accuracy can be directly estimated by comparing the exact and the effective bath hybridization functions (Fig. 2). Also, no often unreliable analytical continuation is required. At the heart of the method lies a solution of the nonequilibrium DMFT impurity problem, which can be seen as a generalization of the exact-diagonalization (ED) approach, widely used in the equilibrium case [16]. However, a crucial difference with respect to conventional DMFT-ED is the fact that here the effective impurity model describes an infinite system and, thus, displays a continuous spectrum.

In ED-based equilibrium DMFT [16], a certain number of noninteracting bath sites is introduced in order to fit the bath hybridization function required by the the selfconsistency condition. The maximum number of bath sites is limited by the exponential increase of the many-body Hilbert space. In equilibrium, the fit is carried out in imaginary (Matsubara) frequency space, where functions 
are smooth, in contrast to real frequency. There are several difficulties in this approach when trying to extend it to nonequilibrium steady states. (i) Due to the finite number of bath sites, a stationary solution of the impurity problem will always produce some equilibrium selfenergy. Besides the fact that this may be questionable, it is not clear which chemical potential or temperature should be used for the impurity problem. (ii) Due to the finite system, the bath spectrum is discontinuous, so that a fit in real frequencies becomes problematic. Unfortunately, there is no Matsubara Green's function in nonequilibrium, so this poses a serious problem.

The alternative presented in this Letter consists in replacing the DMFT impurity Hamiltonian with an effective one that is solvable by ED but at the same time describes a truly infinite system. This is obtained by connecting the interacting impurity to a moderate number of bath sites that, in turn, are attached to Markovian reservoirs; see below for details. The exact bath spectral function is smoothly obtained in the (ideal) limit of an infinite number of bath sites. The action of such Markovian baths on the reduced density matrix of the system consisting of the other bath sites and of the impurity is described by the Lindblad quantum master equation [50]. The latter can be readily solved by diagonalizing the Lindbladian within the many-body "super-Fock" space of reduced density matrices of the system. Its solution determines both the retarded and Keldysh impurity Green's function as well as the selfenergy. The latter is used in the DMFT loop to obtain the new bath hybridization function, which is fitted by new bath parameters.

In order to illustrate the approach, we apply it to a simple model describing a heterojunction consisting of a correlated interface $(c)$ sandwiched between two metallic leads $\alpha=l, r$ (see Fig. 1). Experimentally, such a setup has been recently explored where the correlated layer was realized by a $\mathrm{V}_{2} \mathrm{O}_{3}$ microfilm that is coupled to Au leads [51].

Before a certain time $\tau<\tau_{0}$, the three regions $c, l$, and $r$ are disconnected and in equilibrium at different chemical potentials $\mu_{c}, \mu_{l}$, and $\mu_{r}$, respectively. This amounts to applying a bias voltage $\Phi=\mu_{l^{-}} \mu_{r}$ between the leads.

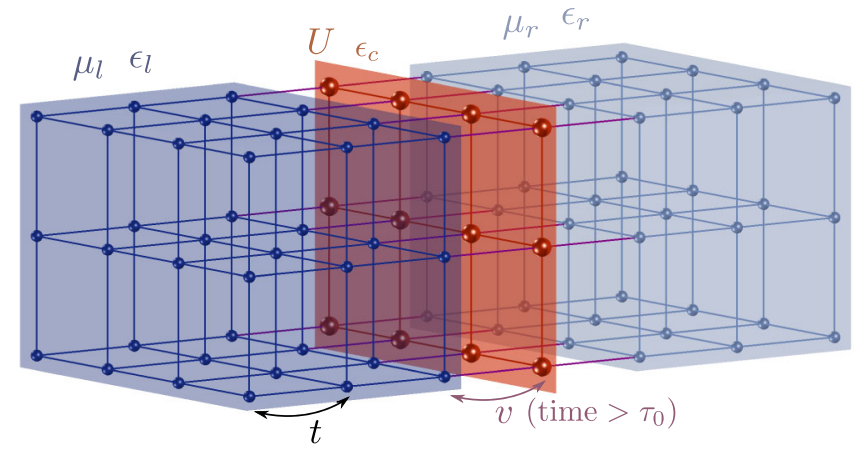

FIG. 1 (color online). Schematic representation of the system at study.
The central region, lying on the $x-y$ plane, is described by a single-band Hubbard layer on a square lattice with onsite interaction $U$, on-site energy $\varepsilon_{c}$, and nearest-neighbor hopping $t$. The leads consist of two half-infinite cubic lattices described by a nearest-neighbor noninteracting tight-binding model with hopping that we take as unit of the energy $t=1$ and on-site energies $\varepsilon_{\alpha}$. We restrict for simplicity to the particle-hole symmetric case for which $\varepsilon_{c}=-U / 2, \mu_{r}=-\mu_{l}$, and $\varepsilon_{r}=-\varepsilon_{l}$. Finally, we take $\mu_{\alpha}=\varepsilon_{\alpha}$, which corresponds to having the same electron densities in the two leads. A related nonequilibrium model has been treated in DMFT within perturbative impurity solvers in Refs. [25,31].

Starting at $\tau=\tau_{0}$, a nearest-neighbor hopping $v$ is switched on between the central region and the leads. After a sufficiently long time, a steady state is reached, provided no trapped surface states occur. Nonequilibrium properties, in general, and nonlinear transport, in particular, can quite generally be addressed in the framework of the Keldysh Green's function approach [26-29,52]. Here, we adopt the notation (see, e.g., Ref. [29]) where the (underlined) Keldysh Green's function is a $2 \times 2$ matrix containing the retarded $\left(G^{R}\right)$, advanced $\left(G^{A}\right)$, and Keldysh $\left(G^{K}\right)$ components. In a steady state, these depend on a single frequency only. The system is translation invariant in the direction parallel to the layer, which we denote as II. Accordingly, we can write Dyson's equation for the layer (c) Green's function $\underline{G}\left(\mathbf{k}_{\|}, \omega\right), \mathbf{k}_{\|}$being the $\|$momentum [52] as

$\underline{G}\left(\mathbf{k}_{\|}, \omega\right)^{-1}=\underline{g}_{0}^{-1}(\omega)-\sum_{\alpha=l, r} v^{2} \underline{g}_{\alpha}\left(\mathbf{k}_{\|}, \omega\right)-\underline{\Sigma}\left(\mathbf{k}_{\|}, \omega\right)$.

Here, $\underline{\Sigma}\left(\mathbf{k}_{\|}, \omega\right)$ is the self-energy; $\underline{g}_{0}$ is the $v=0, U=0$ layer Green's function; and $g_{\alpha}\left(\mathbf{k}_{\|}, \omega\right)$ are the $v=0$ lead Green's functions on the first lead layers. Their retarded and Keldysh components are readily obtained analytically in terms of the Green's function of a half-infinite tightbinding chain.

Within DMFT, one approximates the self-energy by a local, i.e., $\mathbf{k}_{\|}$-independent, $\underline{\Sigma}(\omega)$ that is determined by solving a quantum impurity model with the same interaction $U$ embedded in a self-consistently determined bath [16]. The latter is completely specified by the bath hybridization function $\underline{\Delta}(\omega)$ that is determined selfconsistently by requiring that the Green's function of the impurity $\underline{G}_{\mathrm{IMP}}(\omega)=\left[\underline{G}_{0}(\omega)^{-1}-\underline{\Sigma}(\omega)\right]^{-1}$ be equal to the local Green's function of the layer (cf. Refs. [16,21,25]) $\underline{G}_{\mathrm{LOC}}(\omega)=\int \frac{d \mathbf{k}_{\|}}{(2 \pi)^{2}} \underline{G}\left(\mathbf{k}_{\|}, \omega\right)$, where $\underline{G}\left(\mathbf{k}_{\|}, \omega\right)$ is given by (1) with $\underline{\Sigma}\left(\mathbf{k}_{\|}, \omega\right)=\underline{\Sigma}(\omega)$, as obtained by the solution of the impurity problem. Here, $\underline{G}_{0}$ is the "Weiss" bare Green's function of the impurity model defined as $\underline{G}_{0}(\omega)^{-1}=\underline{g}_{0}(\omega)^{-1}-\underline{\Delta}(\omega)$.

The solution of the impurity problem is in fact the DMFT bottleneck. The usual (equilibrium) DMFT-ED 
procedure consists in approximating the effect of the total bath hybridization function $\Delta(\omega)$ by an "effective" bath with a finite number $N_{b}$ of bath sites. Quite generally, in equilibrium, one carries out some fit to the bath hybridization function in Matsubara space. As discussed above, this is not appropriate in a nonequilibrium steady state. In this Letter, we present a different approach: In addition to a certain (even) number $N_{b}$ of bath sites that we more conveniently connect to the impurity in the form of two chain segments, we include two Markovian baths that represent a particle reservoir and sink, respectively. Their role is to compensate for the "missing" part of the infinite chain that would be necessary to exactly reproduce the desired $\underline{\Delta}(\omega)$. The bath parameters, i.e., the hopping and on-site energies of the bath sites, as well as the Lindblad coefficients (see below) of the Markovian baths, are then fitted to $\Delta(\omega)$. More specifically, we minimize the cost function $\int d \omega \sum_{x=R, K}\left\{\operatorname{Im}\left[\Delta^{x}(\omega)-\Delta_{\text {eff }}^{x}(\omega)\right]\right\}^{2}$, where $\underline{\Delta}(\omega)$ is obtained from $\underline{G}_{\text {LOC }}$ via

$$
\underline{\Delta}(\omega)=\underline{g}_{0}(\omega)^{-1}-\underline{G}_{\mathrm{LOC}}(\omega)^{-1}-\underline{\Sigma}(\omega),
$$

while $\Delta_{\text {eff }}$ is the bath hybridization function produced by the effective bath (bath sites + Markovian baths). An important aspect is that, although the (outermost) baths are Markovian, their effect on the impurity site is nonMarkovian due to the presence of the intermediate bath sites. This can be seen, for example, in the spectrum of $\underline{\Delta}_{\text {eff }}$ in Fig. 2, which in the Markovian case would be a constant. Furthermore, upon increasing the number $N_{b}$ of intermediate bath sites, the effect of the Markovian bath becomes weaker and one is expected to approach the exact result $\underline{\Delta}_{\text {eff }}(\omega)=\underline{\Delta}(\omega)$ for large $N_{b}$.

We now specify the effective bath more in detail. This consists of an Hamiltonian for the "system" (a chain of impurity + bath sites)

$$
H=\sum_{n, m, \sigma} E_{n, m} c_{n \sigma}^{\dagger} c_{m \sigma}+U c_{0 \uparrow}^{\dagger} c_{0 \uparrow} c_{0 \downarrow}^{\dagger} c_{0 \downarrow},
$$

in the usual notation, where 0 is the impurity site and $n=-1, \ldots,-l(n=1, \ldots, l)$ are the left (right) bath sites.
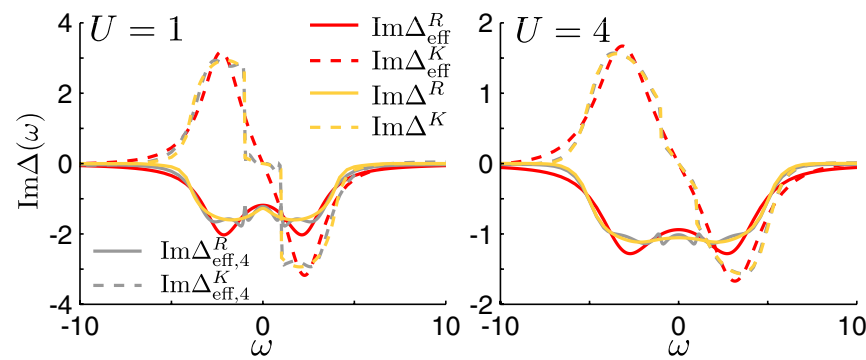

FIG. 2 (color online). Imaginary part of the DMFT and effective bath hybridization functions [retarded $(R)$ and Keldysh $(K)$ components] for $\Phi=2, v^{2}=0.1$, and two different values of $U$. We also plot the effective bath hybridization function $\left(\underline{\Delta}_{\text {eff, } 4}\right)$ obtained with $N_{b}=4$.
Here, $E_{0,0}=\varepsilon_{c}$ and the bath energies $E_{n, n}, n \neq 0$, as well as the hoppings $E_{n, m}, n \neq m$, are fit parameters [53], whereby one can restrict to the nearest neighbors $E_{n, n \pm 1}$. The effect of the Markovian baths is expressed in terms of the Lindblad quantum master equation that controls the time $(\tau)$ dependence of the reduced density matrix $\rho$ of the system [50]: $\frac{d}{d \tau} \rho=\mathcal{L} \rho$, where $\mathcal{L}=\mathcal{L}_{H}+\mathcal{L}_{b}$ and $\mathcal{L}_{H} \rho=-i[H, \rho]$. The dissipator $\mathcal{L}_{b}$ has the form

$$
\begin{aligned}
\mathcal{L}_{b} \rho \equiv & 2 \sum_{n, m}\left[\Gamma_{n, m}^{(1)}\left(c_{n \sigma} \rho c_{m \sigma}^{\dagger}-\frac{1}{2}\left\{\rho, c_{m \sigma}^{\dagger} c_{n \sigma}\right\}\right)\right. \\
& \left.+\Gamma_{n, m}^{(2)}\left(c_{n \sigma}^{\dagger} \rho c_{m \sigma}-\frac{1}{2}\left\{\rho, c_{m \sigma} c_{n \sigma}^{\dagger}\right\}\right)\right],
\end{aligned}
$$

with real, symmetric, and positive definite Lindblad matrices $\Gamma_{n, m}^{(i)}$. At first sight, one would assume a "source" bath attached to the leftmost and a "sink" bath to the rightmost site. However, the accuracy improves considerably if one allows all $\Gamma_{n, m}^{(i)}$ to be nonzero parameters to fit $\underline{\Delta}$.

As discussed in detail in Refs. [54-57], the open-system problem describing the effective bath can be mapped onto a super-Hamiltonian acting on a superfermion space with twice as many degrees of freedom (i.e., "orbitals"). This many-body super-Hamiltonian, corresponding to $i \mathcal{L}$, which is non-Hermitian, can be diagonalized by conventional methods within the super-Hilbert space. Quite generally, $\mathcal{L}$ has a unique eigenvector with eigenvalue 0 that corresponds to the steady-state density matrix $\rho_{S S}$. All other eigenvalues have a negative real part, corresponding to decaying terms. With the same formalism and exploiting the quantum regression theorem [58], one can evaluate correlation functions $C_{A B}(\tau)=\operatorname{tr} A(\tau) B(0) \rho_{S S}$ of any pair of system operators $A$ and $B$, and thus the required impurity self-energy $\underline{\Sigma}(\omega)$ [59].

The noninteracting Green's function for the effective system + bath is necessary in order to extract $\Sigma(\omega)$ and the bath hybridization function $\underline{\Delta}_{\text {eff }}$. This can be easily obtained [60] by observing that the Markovian baths can be exactly represented by two noninteracting fermionic baths in the wide-band limit with chemical potentials $\pm \infty$. By taking into account the relation between the matrices $\Gamma$ and the parameters of this bath [61], one obtains for the noninteracting system Green's function (boldface objects represent matrices with indices corresponding to system sites $n)\left(\mathbf{G}_{0}^{-1}\right)^{R}=\omega \mathbf{I}-\mathbf{E}+i\left(\boldsymbol{\Gamma}^{(1)}+\boldsymbol{\Gamma}^{(2)}\right)$ and $\left(\mathbf{G}_{0}^{-1}\right)^{K}=2 i\left(\boldsymbol{\Gamma}^{(1)}-\boldsymbol{\Gamma}^{(2)}\right)$.

The DMFT self-consistency loop consists in (i) starting from some initial values of the variational parameters $E_{n, m}$ and $\Gamma_{n, m}^{(i)}$, (ii) solving the impurity problem via the approach described above and determining $\underline{\Sigma}$, (iii) evaluating $\underline{G}_{\mathrm{LOC}}(\omega)$ and $\underline{\Delta}(\omega)$ [from (2)], (iv) determining new values of the parameters $E_{n, m}$ and $\Gamma_{n, m}^{(i)}$ by minimizing the cost function, and, finally, (v) using these new parameters to repeat the procedure from (i) until the parameter values converge. Of course, there is an intrinsic inaccuracy that, 
for a fixed number of bath sites, cannot be reduced and is due to the error in the fit of $\Delta(\omega)$ by a finite number of parameters. In principle, this can be systematically improved by increasing the number of bath sites. Of course, this is limited by the exponential increase of the Hilbert space, which, in this case, is even faster due to the fact that the number of degrees of freedom of the superfermion space is twice the one of the fermion space, so this makes the effort more difficult than in ordinary ED for the same number of bath sites. One should, however, observe that the number of fit parameters is larger than in the case with "simple" ED without a Markovian bath [62].

We have used here an effective bath containing $N_{b}=2$ sites. Still, by taking all possible parameters into account, i.e., allowing, for example, all matrix elements of the $\Gamma$ matrices to be nonzero (within the constraints imposed by symmetries), this gives eight independent fitting parameters. In "simple" ED, one would have only two in the particle-hole symmetric case [63]. We take model parameters [64] $v^{2}=0.1, t=1$, and the leads are fixed to zero temperature. In Fig. 2, we show the result of the fit to the bath hybridization function for two values of $U$ and bias voltage $\Phi=2$. As one can see, already with this small number of bath sites, the fit is quite good. Moreover, the structure of $\underline{\Delta}_{\text {eff }}$ is clearly non-Markovian, as expected. For comparison, we also plot the result of the fit to the bath hybridization function obtained with $N_{b}=4$ bath sites. This shows a considerable improvement. The quality of the fit can be also inferred by directly plotting the local and the impurity Green's functions in Fig. 3 for two different values of $U$ and $\Phi=2$.

The bias voltage induces a steady-state current. The expression for the current density $j$ (current per square plaquette) is obtained straightforwardly in terms of the

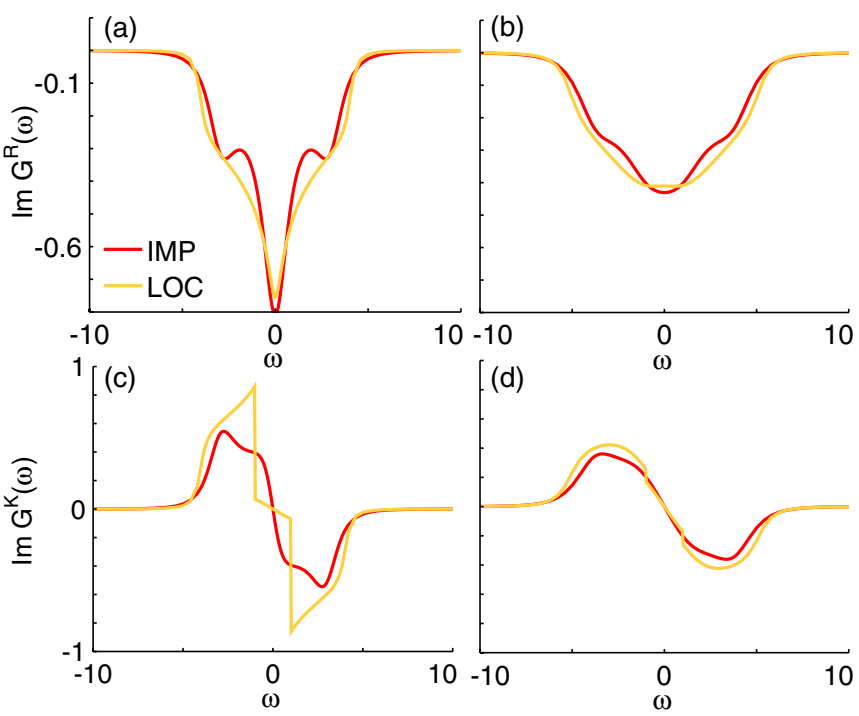

FIG. 3 (color online). Imaginary part of the impurity and local Green's function for $U=1$ (left) and $U=4$ (right) and $\Phi=2$. layer Green's function and the $v=0$ lead Green's functions (see, e.g., Ref. [52]) as

$$
\begin{aligned}
j= & v^{2} \int_{B Z} \frac{d \mathbf{k}_{\|}}{(2 \pi)^{2}} \int \frac{d \omega}{2 \pi} \operatorname{Re}\left[G^{R}\left(\mathbf{k}_{\|}, \omega\right) g_{l}^{K}\left(\mathbf{k}_{\|}, \omega\right)\right. \\
& \left.+G^{K}\left(\mathbf{k}_{\|}, \omega\right) g_{l}^{A}\left(\mathbf{k}_{\|}, \omega\right)\right] .
\end{aligned}
$$

This is plotted in Fig. 4 as a function of bias voltage. The current, as expected, decreases with increasing $U$ for smaller biases. At larger $\Phi$, the behavior is opposite since $j$ extends over a range of voltages that increases with increasing $U$. While at $U=0$, a particle going through the interface conserves $\mathbf{k}_{\|}$, and thus the current goes to zero at a bias voltage equal to the one-dimensional (z-direction) bandwidth and the scattering at nonzero $U$ mixes $\mathbf{k}_{\|}$and thus broadens the bandwidth of possible final states. In Fig. 4, we plot the scaled current $j / v^{2}$ as a function of bias voltage for different values of $U$, as the conductance is expected to behave as $v^{2}$ in a conductor, while it is suppressed $\left(\propto v^{4}\right)$ in a gapped system. The crossing of the curves around $U \sim 4$ is a signal of the nearby equilibrium metal-insulator transition. However, this should be seen only as indicative, as the curves are taken at a relatively high bias.

In conclusion, we have introduced a versatile method to deal with strongly correlated systems out of equilibrium within dynamical mean-field theory. The DMFT selfconsistent bath is approximated by an effective one

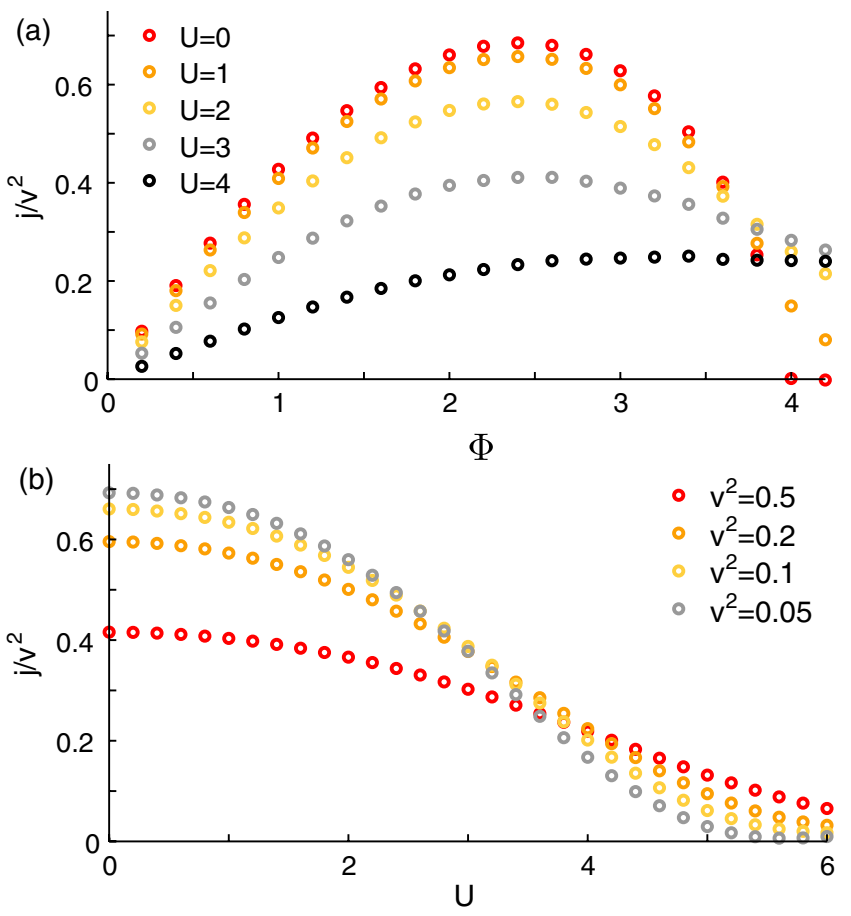

FIG. 4 (color online). Scaled current density $j / v^{2}$ as a function of (a) the bias voltage $\Phi$, for different values of $U$ and $v^{2}=0.1$, and (b) the interaction $U$, for different values of the hybridization $v$ and $\Phi=2$. 
consisting of a small number of sites coupled to a Markovian bath environment. The steady-state and Green's functions of the effective system are solved by the ED of the corresponding Lindblad equation. The approach is particularly appropriate to deal directly with the steady state, without the need to consider full-time evolution. Nevertheless, it should be straightforward, although computationally more demanding, to deal with time-dependent problems, e.g., to describe pump-probe processes.

The accuracy of the effective bath to reproduce the DMFT one obviously depends on the number of bath sites, which is limited by the exponential increase of the "super"-Hilbert space. Improvements can possibly go along solving the Lindblad problem in the "smaller" ordinary fermion space in combination with quantum trajectory methods [65-67] and/or by density matrix renormalization group approaches [68-70].

The approach illustrated here for a simple but experimentally relevant [51] model can be extended straightforwardly to a number of other physically relevant systems, including multilayer semiconducting heterostructures, ultracold atoms, and correlated coupled-cavity arrays featuring driving and dissipation and molecular contacts, and can be used to study nonequilibrium quantum phase transitions in these systems. Extension to a nonlocal selfenergy, as in cluster DMFT or in nonequilibrium variational or perturbative cluster approaches [71-73], is also an interesting development.

We acknowledge illuminating discussions with S. Diehl. This work is partly supported by the Austrian Science Fund (FWF) Grants No. F4103-N13, No. J3361-N20, and No. P24081-N16.

*arrigoni@tugraz.at

[1] M. Raizen, C. Salomon, and Q. Niu, Phys. Today 50, No. 7, 30 (1997).

[2] D. Jaksch, C. Bruder, J. I. Cirac, C. W. Gardiner, and P. Zoller, Phys. Rev. Lett. 81, 3108 (1998).

[3] M. Greiner, O. Mandel, T. Esslinger, T. W. Hänsch, and I. Bloch, Nature (London) 415, 39 (2002).

[4] M. Hartmann, F. Brandão, and M. Plenio, Laser Photonics Rev. 2, 527 (2008).

[5] I. Zutic, J. Fabian, and S. D. Sarma, Rev. Mod. Phys. 76, 323 (2004)

[6] L. L. Bonilla and H. T. Grahn, Rep. Prog. Phys. 68, 577 (2005).

[7] S. Iwai, M. Ono, A. Maeda, H. Matsuzaki, H. Kishida, H. Okamoto, and Y. Tokura, Phys. Rev. Lett. 91, 057401 (2003).

[8] A. Cavalleri, T. Dekorsy, H. H.W. Chong, J. C. Kieffer, and R. W. Schoenlein, Phys. Rev. B 70, 161102 (2004).

[9] L. Perfetti, P. A. Loukakos, M. Lisowski, U. Bovensiepen, H. Berger, S. Biermann, P. S. Cornaglia, A. Georges, and M. Wolf, Phys. Rev. Lett. 97, 067402 (2006).
[10] D. Fausti, R. I. Tobey, N. Dean, S. Kaiser, A. Dienst, M. C. Hoffmann, S. Pyon, T. Takayama, H. Takagi, and A. Cavalleri, Science 331, 189 (2011).

[11] A. Mitra, S. Takei, Y. B. Kim, and A. J. Millis, Phys. Rev. Lett. 97, 236808 (2006).

[12] A. J. Leggett, S. Chakravarty, A. T. Dorsey, M. P. A. Fisher, A. Garg, and W. Zwerger, Rev. Mod. Phys. 59, 1 (1987).

[13] M. A. Cazalilla, Phys. Rev. Lett. 97, 156403 (2006).

[14] P. Calabrese and J. Cardy, J. Stat. Mech. (2007) P06008.

[15] M. Rigol, V. Dunjko, and M. Olshanii, Nature (London) 452, 854 (2008).

[16] A. Georges, G. Kotliar, W. Krauth, and M. J. Rozenberg, Rev. Mod. Phys. 68, 13 (1996).

[17] D. Vollhardt, in Lectures on the Physics of Strongly Correlated Systems, edited by A. Avella and F. Mancini, AIP Conf. Proc. Vol. 1297 (AIP, New York, 2010), p. 339.

[18] W. Metzner and D. Vollhardt, Phys. Rev. Lett. 62, 324 (1989).

[19] V. I. Anisimov, A. I. Poteryaev, M. A. Korotin, A. O. Anokhin, and G. Kotliar, J. Phys. Condens. Matter 9, 7359 (1997)

[20] P. Schmidt and H. Monien, arXiv:cond-mat/0202046.

[21] J. K. Freericks, V. M. Turkowski, and V. Zlatić, Phys. Rev. Lett. 97, 266408 (2006)

[22] J. K. Freericks, Phys. Rev. B 77, 075109 (2008).

[23] A. V. Joura, J. K. Freericks, and T. Pruschke, Phys. Rev. Lett. 101, 196401 (2008).

[24] M. Eckstein, M. Kollar, and P. Werner, Phys. Rev. Lett. 103, 056403 (2009).

[25] S. Okamoto, Phys. Rev. B 76, 035105 (2007).

[26] J. Schwinger, J. Math. Phys. (N.Y.) 2, 407 (1961).

[27] L. V. Keldysh, Sov. Phys. JETP 20, 1018 (1965).

[28] L.P. Kadanoff and G. Baym, Quantum Statistical Mechanics: Green's Function Methods in Equilibrium and Nonequilibrium Problems (Addison-Wesley, Redwood City, CA, 1962).

[29] J. Rammer and H. Smith, Rev. Mod. Phys. 58, 323 (1986).

[30] M. Eckstein, M. Kollar, and P. Werner, Phys. Rev. B 81, 115131 (2010).

[31] S. Okamoto, Phys. Rev. Lett. 101, 116807 (2008).

[32] N. Tsuji, T. Oka, and H. Aoki, Phys. Rev. B 78, 235124 (2008).

[33] N. Tsuji, T. Oka, and H. Aoki, Phys. Rev. Lett. 103, 047403 (2009).

[34] P. Mehta and N. Andrei, Phys. Rev. Lett. 96, 216802 (2006).

[35] F. B. Anders, Phys. Rev. Lett. 101, 066804 (2008).

[36] Y. Meir and N. S. Wingreen, Phys. Rev. Lett. 68, 2512 (1992).

[37] H. Schoeller and G. Schön, Phys. Rev. B 50, 18436 (1994).

[38] A. Rosch, J. Paaske, J. Kroha, and P. Wölfle, J. Phys. Soc. Jpn. 74, 118 (2005).

[39] H. Schoeller, Eur. Phys. J. Special Topics 168, 179 (2009).

[40] S. R. White and A. E. Feiguin, Phys. Rev. Lett. 93, 076401 (2004).

[41] A. J. Daley, C. Kollath, U. Schollwöck, and G. Vidal, J. Stat. Mech. (2004) P04005.

[42] F. B. Anders and A. Schiller, Phys. Rev. Lett. 95, 196801 (2005). 
[43] S. Kehrein, Phys. Rev. Lett. 95, 056602 (2005).

[44] R. Gezzi, T. Pruschke, and V. Meden, Phys. Rev. B 75, 045324 (2007).

[45] S. G. Jakobs, V. Meden, and H. Schoeller, Phys. Rev. Lett. 99, 150603 (2007).

[46] C. Jung, A. Lieder, S. Brener, H. Hafermann, B. Baxevanis, A. Chudnovskiy, A. Rubtsov, M. Katsnelson, and A. Lichtenstein, Ann. Phys. (Amsterdam) 524, 49 (2012).

[47] J.E. Han, Phys. Rev. B 73, 125319 (2006).

[48] J.E. Han and R. J. Heary, Phys. Rev. Lett. 99, 236808 (2007).

[49] A. Dirks, P. Werner, M. Jarrell, and T. Pruschke, Phys. Rev. E 82, 026701 (2010).

[50] H.-P. Breuer and F. Petruccione, The Theory of Open Quantum Systems (Oxford University Press, Oxford, England, 2009).

[51] S. Guénon, S. Scharinger, S. Wang, J. G. Ramírez, D. Koelle, R. Kleiner, and I. K. Schuller, arXiv:1210.6648.

[52] H. Haug and A.-P. Jauho, Quantum Kinetics in Transport and Optics of Semiconductors (Springer, Heidelberg, 1998).

[53] In general, $E_{0,0}$ can be taken as a fit parameter too, but in the present case it is fixed by particle-hole symmetry.

[54] M. Schmutz, Z. Phys. B 30, 97 (1978).

[55] U. Harbola and S. Mukamel, Phys. Rep. 465, 191 (2008).

[56] A. A. Dzhioev and D. S. Kosov, J. Chem. Phys. 134, 044121 (2011).

[57] T. Prosen, New J. Phys. 10, 043026 (2008).

[58] H. J. Carmichael, Statistical Methods in Quantum Optics: Master Equations and Fokker-Planck Equations, Texts and Monographs in Physics Vol. 1 (Springer, Singapore, 2002).

[59] See Supplemental Material at http://link.aps.org/ supplemental/10.1103/PhysRevLett.110.086403 for details of the solution of the impurity problem.

[60] Alternatively and equivalently, the problem can be solved by the "third quantization" approach of Ref. [57].

[61] Details will be given elsewhere.

[62] Roughly speaking, this is due to the fact that each parameter $E_{n, m}$ of the bare system becomes effectively complex, and we can use its real and imaginary part for the fit.

[63] We must fit two functions, though, $\Delta^{R}$ and $\Delta^{K}$. Still, the number of parameters per function is twice as large.

[64] These are the physical parameters, not to be confused with the self-consistent parameters $E$ and $\Gamma$.

[65] C. W. Gardiner and P. Zoller, Quantum Noise (Springer, Berlin, 2000).

[66] H. J. Carmichael, An Open Systems Approach to Quantum Optics (Springer, Berlin, 1993).

[67] A. J. Daley, J.M. Taylor, S. Diehl, M. Baranov, and P. Zoller, Phys. Rev. Lett. 102, 040402 (2009).

[68] S. R. White, Phys. Rev. Lett. 69, 2863 (1992).

[69] F. Verstraete, J. J. García-Ripoll, and J. I. Cirac, Phys. Rev. Lett. 93, 207204 (2004).

[70] T. Prosen and M. Znidaric, J. Stat. Mech. (2009) P02035.

[71] M. Knap, W. von der Linden, and E. Arrigoni, Phys. Rev. B 84, 115145 (2011).

[72] M. Potthoff, Eur. Phys. J. B 32, 429 (2003).

[73] M. Balzer and M. Potthoff, Phys. Rev. B 83, 195132 (2011). 